\title{
Selective amino acid only in vivo NMR: A powerful tool to follow stress processes
}

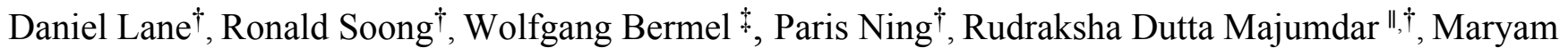
Tabatabaei Anaraki ${ }^{\dagger}$, Hermann Heumann ${ }^{\S}$, Marcel Gundy ${ }^{\S}$, Holger Bönisch ${ }^{\S}$, Yalda Liaghati Mobarhan ${ }^{\dagger}$, Myrna J. Simpson ${ }^{\dagger}$, André J. Simpson*,†

$\dagger$ Environmental NMR Centre, Department of Physical and Environmental Science, University of Toronto, 1265 Military Trail, Toronto, ON, Canada, M1C 1A4

†ruker BioSpin GmbH. Silberstreifen 4 Rheinstetten Germany

II Bruker Canada Ltd, 2800 High Point Drive, Milton, Ontario, L9T 6P4

$\S$ Silantes GmbH, München, Germany

*Corresponding Author (andre.simpson@utoronto.ca) 


\section{SUPPORTING INFORMATION}

The Supporting Information contains the following sections:

1. Supporting Figures S1-S2

2. Table S1

3. Amino Acid NMR Pulse Sequence

1. Supporting Figures S1-S2

S- 2 - 

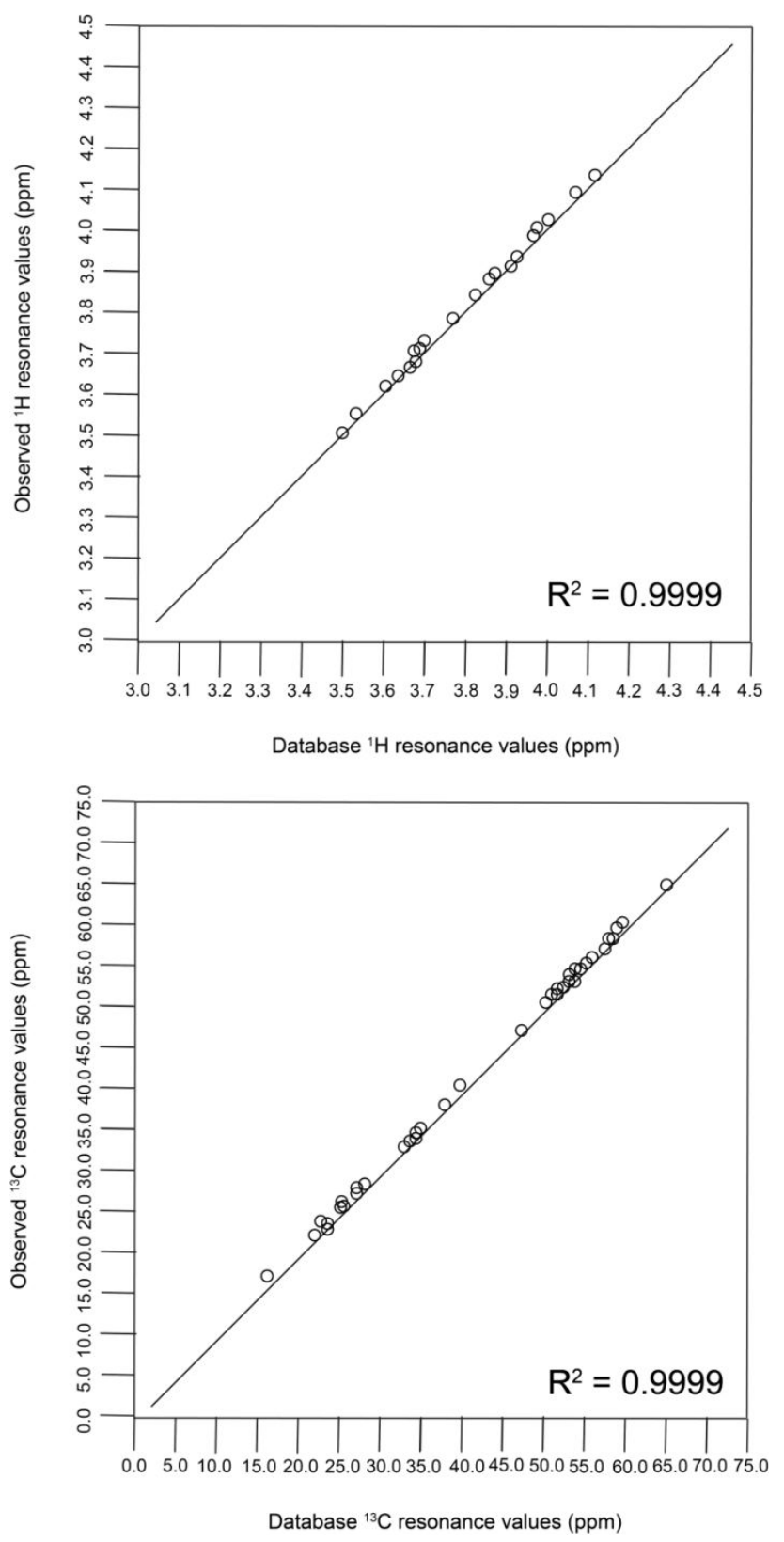

S- 3 - 


\section{Figure S1}

A correlation plot of the $(\mathrm{H}) \mathrm{CbCa}(\mathrm{COCa}) \mathrm{Ha}$ experiment observed chemical shift assignments against those obtained from the Bruker Biofluid Reference Compound Database for all 20 amino acids.

A)

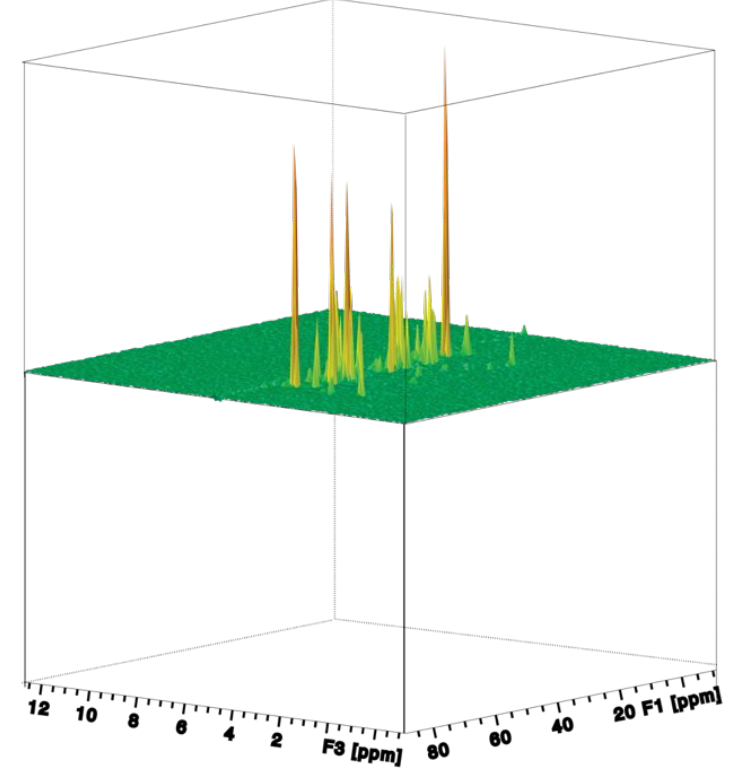

B )

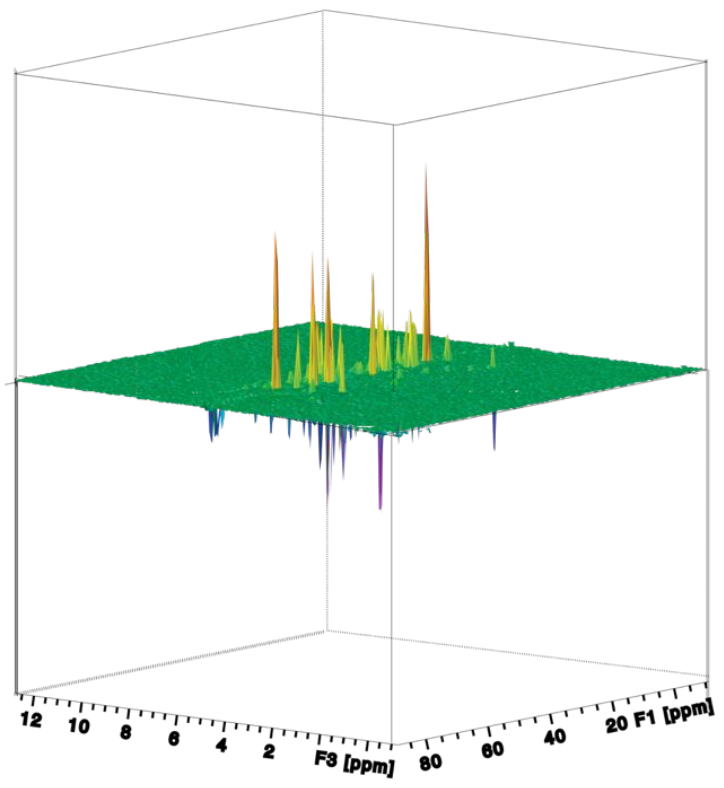

\section{Figure S2}

A $3 \mathrm{D}$ oblique view of in vivo $(\mathrm{H}) \mathrm{CbCa}(\mathrm{COCa}) \mathrm{Ha} \mathrm{NMR}$ spectra of in vivo ${ }^{13} \mathrm{C}$ enriched D. magna acquired with A ) $100 \%$ sampling density and B ) $20 \%$ sampling density using NUS and reconstructed with CS. 


\section{Table S1}

\section{Table S1}

A list of the amino acids that showed a significant statistical change inside D.magna (paired t-test, $\mathrm{p}$-value $<$ 0.05) upon BPA exposure and their corresponding p-value.

\begin{tabular}{|c|c|}
\hline Amino Acid & p-value \\
\hline Alanine & 0.0315 \\
\hline Asparagine & 0.0372 \\
\hline Aspartic Acid & 0.0291 \\
\hline Cysteine & 0.0358 \\
\hline Glycine & 0.0412 \\
\hline Isoleucine & 0.0335 \\
\hline Leucine & 0.0386 \\
\hline Lysine & 0.0364 \\
\hline Phenylalanine & 0.0286 \\
\hline Proline & 0.0425 \\
\hline Serine & 0.0237 \\
\hline Threonine & 0.0441 \\
\hline Tyrosine & 0.0349 \\
\hline Valine & 0.0428 \\
\hline
\end{tabular}

\section{Amino Acid NMR Pulse Sequence}

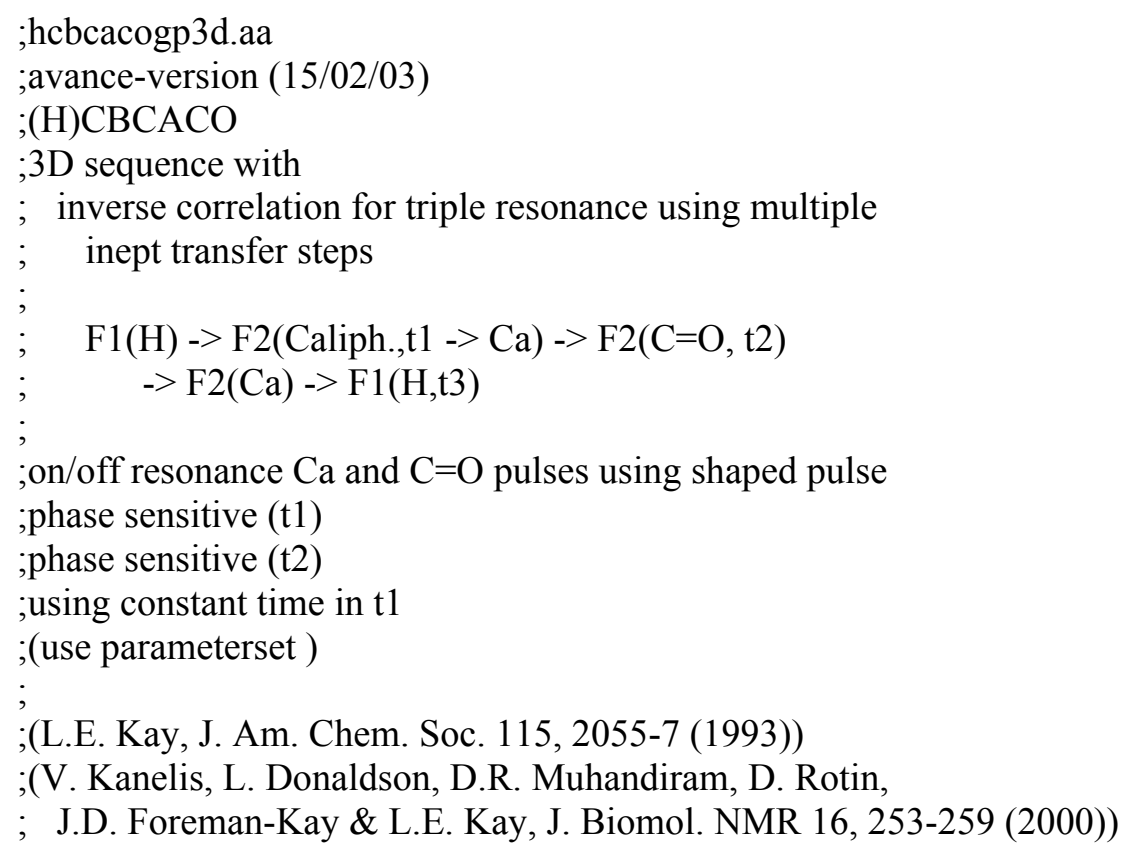


;(A.C. Wang, S. Grzesiek, R. Tschudin, P.J. Lodi \& A. Bax, ; J. Biomol. NMR 5, 376-382 (1995))

;

; $\$$ CLASS=HighRes

;\$DIM=3D

;\$TYPE=

;\$SUBTYPE $=$

; $\$$ COMMENT $=$

prosol relations $=<$ triple $>$

\#include $<$ Avance.incl $>$

\#include $<$ Grad.incl $>$

\#include $<$ Delay.incl $>$

"p2=p1*2"

"p22=p21*2"

"d11=30m"

"d12=20u"

"d $3=1.1 \mathrm{~m} "$

"d4=1.6m"

"d $22=3.6 \mathrm{~m} "$

"d $23=12.0 \mathrm{~m} "$

"d27=4.2m"

"d28=3.6m"

"p30 $=5 \mathrm{~m} "$

"p31=4.4m"

"d0=3u"

"d10 $=3 \mathrm{u} "$

"d20=d28+d0-p14-4u"

"in $0=$ inf $1 / 2 "$

"in10=inf2/4"

"in20=in0"

"DELTA=d10*2+larger(p14,p22)-p14"

"DELTA1=d28-d3-larger(p14,p22)-p2"

"DELTA2=d22-p14-4u"

"DELTA3=d27-p14-4u"

"DELTA4=d27-d3-p2-p14-4u"

"DELTA5=d4-8u" 


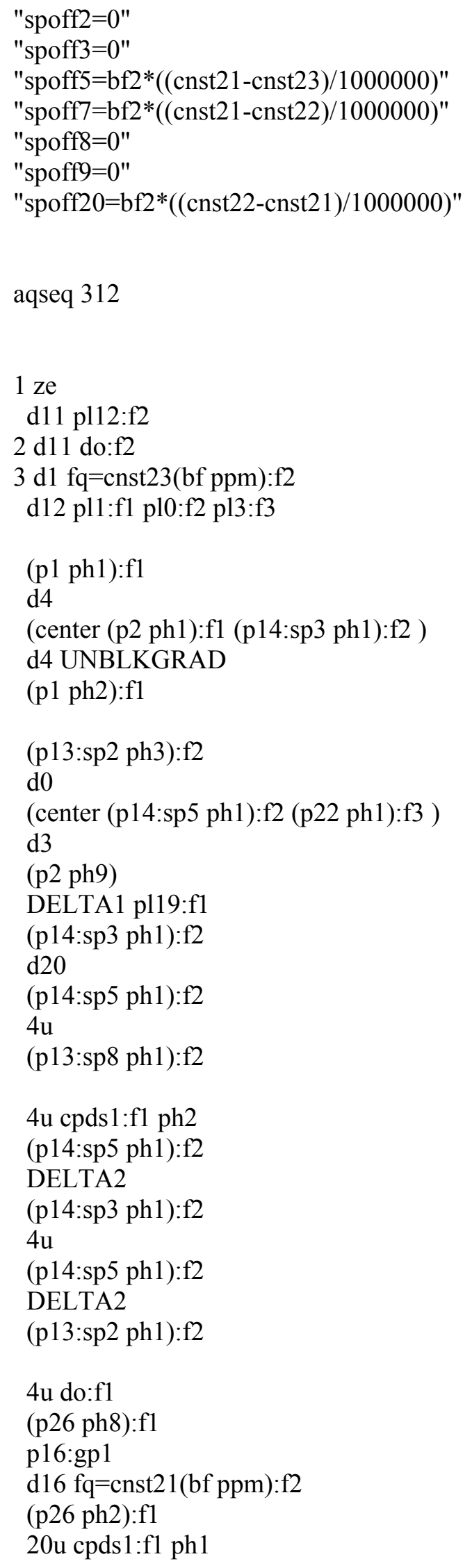




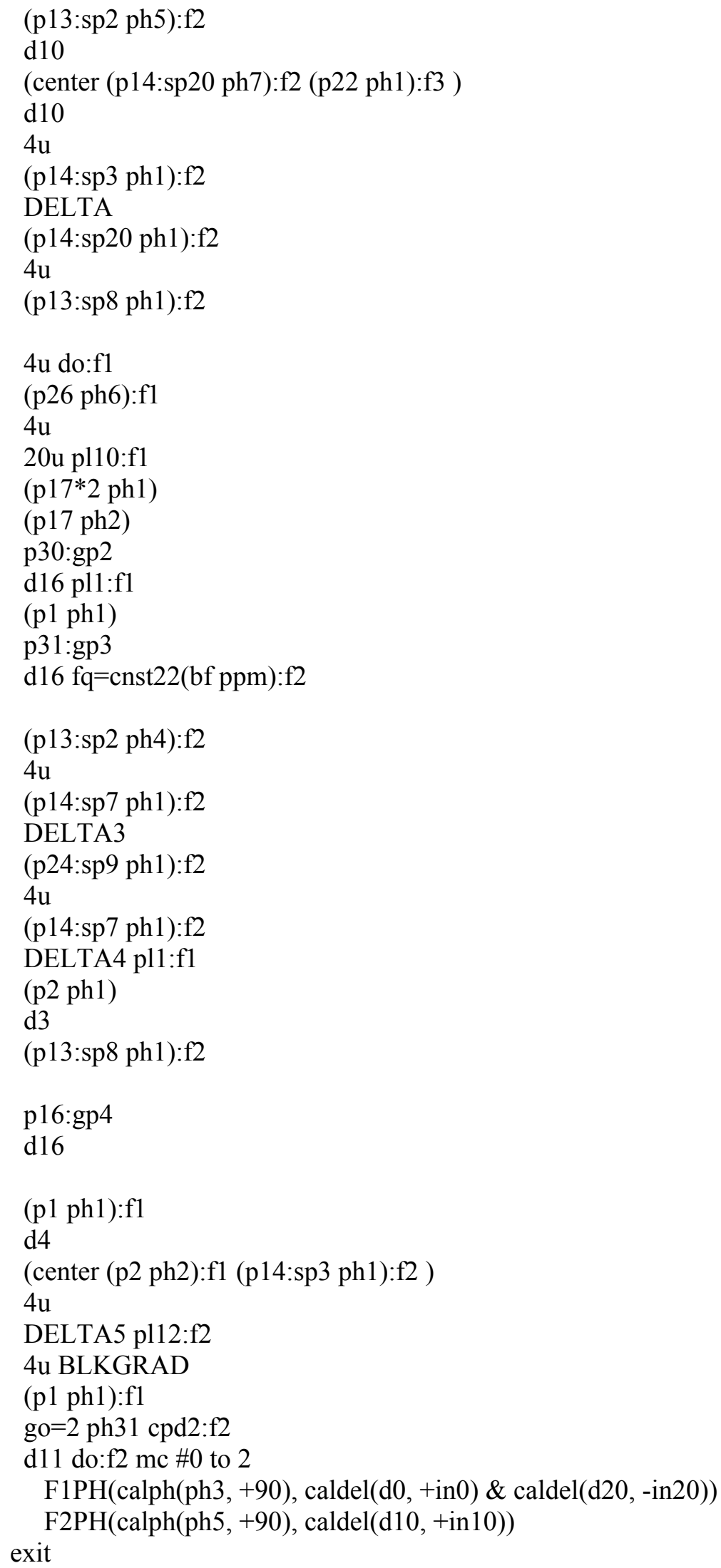


ph1 $=0$

ph $2=1$

$\mathrm{ph} 3=02$

ph4=0 000000000222222222

ph5=0 022

ph6=3

ph7=0 0000222222

ph $8=2$

ph9=0 0222

ph31=0220022020022002

;p10 : 0W

;pl1 : fl channel - power level for pulse (default)

;p13 : f3 channel - power level for pulse (default)

;pl10: f1 channel - power level for TOCSY-spinlock

;pl12: f2 channel - power level for CPD/BB decoupling

;pl19: f1 channel - power level for CPD/BB decoupling

;sp2 : f2 channel - shaped pulse 90 degree (on resonance)

;sp3 : f2 channel - shaped pulse 180 degree (on resonance)

;sp5 : f2 channel - shaped pulse 180 degree $(\mathrm{C}=\mathrm{O}$ off resonance $)$

;sp7 : f2 channel - shaped pulse 180 degree $(\mathrm{C}=\mathrm{O}$ off resonance)

;sp8 : f2 channel - shaped pulse 90 degree (on resonance)

; for time reversed pulse

;sp9 : f2 channel - shaped pulse 180 degree (Ca on resonance)

; $\quad$ sp9 requires higher selectivity than $\mathrm{sp} 3$ (Ca only)

;sp20: f2 channel - shaped pulse 180 degree (Ca off resonance)

;p1 : f1 channel - 90 degree high power pulse

;p2 : f1 channel - 180 degree high power pulse

;p13: f2 channel - 90 degree shaped pulse

;p14: f2 channel - 180 degree shaped pulse

;p16: homospoil/gradient pulse

[1 $\mathrm{msec}]$

;p17: f1 channel - trim pulse

[2.5 $\mathrm{msec}]$

;p21: f3 channel - 90 degree high power pulse

;p22: f3 channel - 180 degree high power pulse

;p24: f2 channel - 180 degree shaped pulse (sp9)

;p26: f1 channel - 90 degree pulse at pl19

;p30: gradient pulse 2

;p31: gradient pulse 3

; 0 : incremented delay (F1 in 3D)

[5 msec]

[4.4 $\mathrm{msec}]$

; 11 : relaxation delay; $1-5 * \mathrm{~T} 1$

;d3 : $1 /(6 \mathrm{~J}(\mathrm{HCa}))$

;d4 : 1/(4J(HCa))

;d10: incremented delay (F2 in $3 \mathrm{D})$

;d11: delay for disk I/O

;d12: delay for power switching

[3 usec]

[1.1 msec]

[1.6 msec]

[3 usec]

[30 msec]

;d16: delay for homospoil/gradient recovery

;d20: decremented delay $(\mathrm{F} 1$ in $3 \mathrm{D})=\mathrm{d} 28+\mathrm{d} 0-\mathrm{p} 14-4 \mathrm{u}$

;d22: $1 /\left(4 \mathrm{~J}^{\prime}(\mathrm{COCa})\right.$

;d23: $1 /(4 \mathrm{~J}(\mathrm{NCO})$

[3.6 $\mathrm{msec}]$

;d27: 1/(4J(COCa)

[12 $\mathrm{msec}]$

[4.2 $\mathrm{msec}]$ 
;d28: $1 /(8 \mathrm{~J}(\mathrm{CaCb})) \quad$ [3.6 msec]

;cnst21: CO chemical shift (offset, in ppm)

;cnst22: Calpha chemical shift (offset, in ppm)

;cnst23: Caliphatic chemical shift (offset, in ppm)

;cnst26: Call chemical shift (offset, in ppm) [101 ppm]

;o2p: Caliphatic chemical shift (cnst23)

;inf1: $1 / \mathrm{SW}($ Cali $)=2 * \mathrm{DW}($ Cali $)$

;inf: $1 / \mathrm{SW}(\mathrm{CO})=2 * \mathrm{DW}(\mathrm{CO})$

;in0: $1 /(2 * \mathrm{SW}($ Cali $))=\mathrm{DW}($ Cali $)$

;nd0: 2

;in10: $1 /(4 * \mathrm{SW}(\mathrm{CO}))=(1 / 2) \mathrm{DW}(\mathrm{CO})$

;nd10: 4

;in20: $=$ in 0

;ns: $4 * \mathrm{n}$

;ds: $>=16$

;td1: number of experiments in F1 td1 $\max =2 * \mathrm{~d} 20 /$ in 20

;td2: number of experiments in F2

;FnMODE: States-TPPI (or TPPI) in F1

;FnMODE: States-TPPI (or TPPI) in F2

;cpds1: decoupling according to sequence defined by cpdprg 1 ;cpd2: decoupling according to sequence defined by cpdprg2 ;pcpd1: f1 channel - 90 degree pulse for decoupling sequence ;pcpd2: f2 channel - 90 degree pulse for decoupling sequence

;use gradient ratio: $\quad$ gp $1:$ gp $2:$ gp $3:$ gp 4

; $40: 60: 60:-50$

;for z-only gradients

;gpz1: $40 \%$

;gpz2: $60 \%$

;gpz3: $60 \%$

;gpz4: $-50 \%$

;use gradient files:

;gpnam1: SMSQ10.100

;gpnam2: SMSQ10.100

;gpnam3: SMSQ10.100

;gpnam4: SMSQ10.100

;\$Id: \$ 